\title{
Gyratons on Melvin spacetime
}

\author{
Hedvika Kadlecovâ* and Pavel Krtou讯 \\ Institute of Theoretical Physics, Faculty of Mathematics and Physics, Charles University, \\ $V$ Holešovičkách 2, 18000 Prague 8, Czech Republic
}

(Dated: June 9, 2010)

\begin{abstract}
We present and analyze new exact gyraton solutions of algebraic type II on a background which is a static, cylindrically symmetric Melvin universe of type D. For a vanishing electromagnetic field it reduces to previously studied gyratons on Minkowski background. We demonstrate that the solutions are a member of a more general family of the Kundt spacetimes. We show that the Einstein equations reduce to a set of mostly linear equations on a transverse 2-space and we discuss the properties of polynomial scalar curvature invariants which are generally non-constant but unaffected by the presence of gyratons.

PACS numbers: 04.20.Jb, 04.30.-w, 04.40.Nr
\end{abstract}

\section{INTRODUCTION}

Gyraton solutions represent the gravitational field of a localized matter source with an intrinsic rotation which is moving at the speed of light. Such an idealized ultrarelativistic source can describe a pulse of a spinning radiation beam and it is accompanied by a sandwich or impulsive gravitational wave.

The gravitational fields generated by (nonrotating) light pulses and beams were already studied by Tolman [1] in 1934, who obtained the corresponding solution in the linear approximation of the Einstein theory. Exact solutions of the Einstein-Maxwell equations for such 'pencils of light' were found and analyzed by Peres 2] and Bonnor [3 5]. These solutions belong to a general family of $p p$-waves $[6,7]$.

In the impulsive limit (i.e., for an infinitely thin beam, and for the delta-type distribution of the light-pulse in time), the simplest of these solutions represents the wellknown Aichelburg-Sexl metric [8] which describes the field of a pointlike null particle. Subsequently, more general impulsive waves were found [9-15] (for recent reviews see [16, 17]).

The gyraton solutions are generalization of $p p$-waves which belong to the Kundt class for which the sourcethe beam of radiation - carries not only energy, but also an additional angular momentum. Such spacetimes were first considered by Bonnor in [18], who studied the gravitational field created by a spinning null fluid. In some cases, this may be interpreted as a massless neutrino field [19].

Gyratons on Minkowski background are locally isometric to standard $p p$-waves in the exterior vacuum region, outside the source. The interior region contains a nonexpanding null matter which possesses an intrinsic spin. In general, these solutions are obtained by keeping nondiagonal terms $g_{u i}$ in the Brinkmann form [20] of the $p p$-wave

\footnotetext{
*Electronic address: hedvika.kadlecova@centrum.cz
}

${ }^{\dagger}$ Electronic address: Pavel.Krtous@mff.cuni.cz solution, where $u$ is the null coordinate and $x^{i}$ are orthogonal spatial coordinates. The corresponding energymomentum tensor thus also contains an extra nondiagonal term $T_{u i}=j_{i}$. In four dimensions, the terms $g_{u i}$ can be set to zero locally, using a suitable gauge transformation. However, they can not be globally removed because the gauge invariant contour integral $\oint g_{u i}\left(u, x^{j}\right) \mathrm{d} x^{i}$ around the position of the gyraton is proportional to the nonzero angular momentum density $j_{i}$, which is nonvanishing.

These gyratons were investigated (in the linear approximation) in [21] in higher dimensional flat space, the exact gyraton solutions propagating in an asymptotically flat $D$-dimensional spacetime were further investigated in 22. They proved that the Einstein's equations for gyratons reduce to a set of linear equations in the Euclidean $(D-2)$-dimensional space and showed that the gyraton metrics belong to a class of so called VSI spacetimes for which all polynomial scalar invariants, constructed from the curvature and its covariant derivatives, vanish identically [23]. (For the discussion of spacetimes with nonvanishing but nonpolynomial scalar invariants of curvature, see [24].) Subsequently, charged gyratons in Minkowski space in any dimension were presented in [25].

In [26], the exact gyraton solutions in the asymptotically anti-de Sitter spacetime were found. Namely, they obtained Siklos gyratons which generalize the Siklos family of nonexpanding waves [27] (investigated further in [28]) which belong to the class of spacetimes with constant scalar invariants (CSI spacetimes) 29-32].

Recently, the large class of gyratons on the directproduct spacetimes was found in [33], where we showed that this class of gyratons has similar properties as the previous gyratonic solutions: the Einstein's equations reduce to a set of linear equations in transversal 2-space and these spacetimes belong to the CSI class of spacetimes.

Let us also mention that string gyratons in supergravity were recently found in [34]. Supersymmetric gyraton solutions were also obtained in minimal gauged theory in five dimensions in [35], where the configuration represents a generalization of the Siklos waves with a nonzero angular momentum in anti-de Sitter space. 
The gyratons are important in studies of production of mini black holes or in cosmic ray experiments. The theory of high energy particle collisions was developed in [36, 37] and was applied to gyraton models in [38].

The main purpose of this paper is to further extend the family of gyratonic solutions. In particular, we present new gyraton solutions of algebraic type II, propagating in the Melvin universe.

The Melvin universe [39, 40] is a nonsingular electrovacuum solution with physical properties which are interesting both from a classical and a quantum point of view. The spacetime represents a parallel bundle of magnetic (or electric) flux held together by its own gravitational attraction. The transverse space orthogonal to the direction of the flux has a nontrivial spatial geometry. It was represented in [41] by a suitable embedding diagram which resembles a tall narrow-necked vase. Also in [41, 42] it was shown that no motion can get too far from the axis of symmetry. This aspect is analogous to the attractive effect of a negative cosmological constant in the anti-de Sitter universe.

The Melvin universe was considered as an important model in astrophysical processes related to gravitational collapse because of its stability. It was shown in [40, 41] that the spacetime is surprisingly stable against small radial perturbations and also against large perturbations which are concentrated in a finite region about the axis of symmetry. The asymmetries are radiated away in gravitational and electromagnetic waves [43, 44].

The Melvin universe also appears as a limit in more complicated solutions, in [45] it is obtained as a specific limit of a charged C-metric. The Melvin universe has been generalized to Kaluza-Klein and dilaton theories [46], to nonlinear electrodynamics [47], and has important applications in the study of quantum black hole pair creation in a background electromagnetic field [48 54].

The Melvin universe recently attracted a new interest because it is possible to find gravitational waves in the Melvin universe [43] by an ultrarelativistic boost of the Schwarzschild-Melvin black hole metric [44]. It was shown that these wave solutions are straightforward impulsive limits of a more general class of Kundt spacetimes of type II with an arbitrary profile function, which can be interpreted as gravitational waves propagating on the Melvin spacetime. The gyraton spacetimes investigated in this paper are generalizations of such Kundt waves when their ultrarelativistic source is made of a 'spinning matter'.

The paper is organized as follows. In Section $\amalg$ we review basic information about the Melvin universe which will be useful in the paper. We derive the ansatz for the gyraton metric by a direct transformation from the Kundt form of the metric to Melvin's coordinates. We also review the transverse space geometry of the wave front.

In Section III, we derive the field equations and we simplify them introducing potentials. We discuss the structure of the equations and the gauge freedom of the solutions.

Next, in Section IV] we solve the Einstein-Maxwell equations in the special case of the $\phi$-independent spacetimes, especially with a thin matter source localized on the axis of symmetry.

In Section $\mathrm{V}$ we concentrate on the interpretation of the gyraton solutions. We discuss the properties of the scalar polynomial invariants and the geometric properties of the principal null congruence. We evaluate the curvature tensor in an appropriate tetrad, discuss the Petrov type, the matter content of the spacetime, and properties of the electromagnetic field.

The main results of the paper are summarized in concluding Section VI. Some technical results needed to derive the field equations, spin coefficients and invariants are left to Appendices $\mathrm{A}, \mathrm{B}$, and $\mathrm{C}$.

\section{THE GYRATONS ON THE MELVIN SPACETIME}

\section{A. The Melvin universe}

In this section we briefly review basic properties of the Melvin spacetime [39, 40, 44] which will be useful throughout the paper. The Melvin universe describes an axial electromagnetic field concentrating under influence of its self-gravity. The strength of the electromagnetic field is determined by the parameters $E$ and $B$. In cylindrical coordinates $(t, z, \rho, \phi)$, the metric and the Maxwell tensor read

$$
\begin{gathered}
d s^{2}=\Sigma^{2}\left(-\mathrm{d} t^{2}+\mathrm{d} z^{2}+\mathrm{d} \rho^{2}\right)+\Sigma^{-2} \rho^{2} \mathrm{~d} \phi^{2} \\
F=E \mathrm{~d} z \wedge \mathrm{d} t+B \Sigma^{-2} \rho \mathrm{d} \rho \wedge \mathrm{d} \phi,
\end{gathered}
$$

where

$$
\Sigma=1+\frac{1}{4} \varrho_{\mathrm{EM}} \rho^{2}
$$

The constant $\varrho_{\mathrm{EM}}$ is given by the parameters $E, B$ as $^{1}$

$$
\varrho_{\mathrm{EM}}=\frac{\varkappa \varepsilon_{\mathrm{o}}}{2}\left(E^{2}+B^{2}\right) .
$$

Introducing double null coordinates,

$$
u=\frac{1}{\sqrt{2}}(t-z), \quad v=\frac{1}{\sqrt{2}}(t+z),
$$

we obtain an alternative expression for metric (2.1) and the Maxwell tensor (2.2):

$$
\begin{gathered}
d s^{2}=\Sigma^{2}\left(-2 \mathrm{~d} u \mathrm{~d} v+\mathrm{d} \rho^{2}\right)+\Sigma^{-2} \rho^{2} \mathrm{~d} \phi^{2}, \\
F=E \mathrm{~d} v \wedge \mathrm{d} u+B \Sigma^{-2} \rho \mathrm{d} \rho \wedge \mathrm{d} \phi .
\end{gathered}
$$

\footnotetext{
${ }^{1} \varkappa=8 \pi G$ and $\varepsilon_{\mathrm{o}}$ are gravitational and electromagnetic constants. There are two standard choices of geometrical units: the Gaussian with $\varkappa=8 \pi$ and $\varepsilon_{\mathrm{o}}=1 / 4 \pi$, and SI like with $\varkappa=\varepsilon_{\mathrm{o}}=1$.
} 
The electromagnetic field can be rewritten also in the complex self-dual form, ${ }^{2}$

$$
\mathcal{F}=\mathcal{B}\left(\mathrm{d} v \wedge \mathrm{d} u-i \Sigma^{-2} \rho \mathrm{d} \rho \wedge \mathrm{d} \phi\right)
$$

with the complex constant $\mathcal{B}$ defined as

$$
\mathcal{B}=E+i B
$$

The metric (2.1) resembles a vacuum solution of the Levi-Civita family [6] for a large value of $\rho$. For $\varrho_{\mathrm{EM}}=0$ $(E, B=0)$ spacetime reduces to the Minkowski spacetime in cylindrical coordinates. For $E \neq 0, B=0$ the Maxwell tensor describes an electric field pointing along the $z$-direction, whereas for $E=0, B \neq 0$ we get a purely magnetic field oriented along the $z$-direction.

The metric admits the four Killing vectors

$$
\partial_{t}, \quad \partial_{z}, \quad \partial_{\phi}, \quad z \partial_{t}+t \partial_{z}=v \partial_{v}-u \partial_{u}
$$

which correspond to staticity, cylindrical symmetry, and invariance under a boost transformation. Using the adapted null tetrad $k=\partial_{v}, l=\Sigma^{-2} \partial_{u}$, and $m=\frac{1}{\sqrt{2}}\left(\Sigma^{-1} \partial_{\rho}-i \Sigma \rho^{-1} \partial_{\phi}\right)$, the only non-vanishing components of Weyl and Ricci tensors are

$$
\begin{aligned}
\Psi_{2} & =\frac{1}{2} \varrho_{\mathrm{EM}}\left(-1+\frac{1}{4} \varrho_{\mathrm{EM}} \rho^{2}\right) \Sigma^{-4}, \\
\Phi_{11} & =\frac{1}{2} \varrho_{\mathrm{EM}} \Sigma^{-4} .
\end{aligned}
$$

This demonstrates that the Melvin universe is a nonvacuum solution of the Petrov type $D$, except at points satisfying $\rho=2 / \sqrt{\varrho_{\mathrm{EM}}}$, where the Weyl tensor vanishes. It is interesting to note that the scalar curvature vanishes, $R=0$.

To conclude, the Melvin spacetime belongs to the family of non-expanding, non-twisting type D electrovacuum solutions investigated by Plebański [55]. As a consequence, it also belongs to the general Kundt class [6, 7] which will be important in the following text.

\section{B. The ansatz for the gyratons on Melvin universe}

Gyratons are generalized gravitational waves corresponding to null sources with intrinsic rotation. In general, the gyraton solutions are obtained by adding nondiagonal terms to the metric of the standard gravitational wave solutions, or in other words, by keeping the

\footnotetext{
${ }^{2}$ We follow the notation of [6], namely, $\mathcal{F} \equiv F+i \star F$ is a complex self-dual Maxwell tensor, where the 4-dimensional Hodge dual is $\star F_{\mu \nu}=\frac{1}{2} \varepsilon_{\mu \nu \rho \sigma} F^{\rho \sigma}$. The self-dual condition reads $\star \mathcal{F}=-i \mathcal{F}$. The orientation of the 4-dimensional Levi-Civita tensor is fixed by the sign of the component $\varepsilon_{v u \rho \phi}=\rho \Sigma^{2}$. The energy-momentum tensor of the electromagnetic field is given by $T_{\mu \nu}=\frac{\varepsilon_{0}}{2} \mathcal{F}_{\mu} \rho \overline{\mathcal{F}}_{\nu \rho}$.
}

non-diagonal terms $g_{u i}$ in the standard Kundt metric [6] . Therefore, we derive the ansatz for the gyraton on Melvin spacetime by adding such new terms to the Kundt form of the Melvin metric. It can be explicitly obtained from (2.6) by transformation

$$
v=\Sigma^{-2} r
$$

which leads to

$$
d s^{2}=-2 \mathrm{~d} u \mathrm{~d} r+d s_{\top}^{2}+2 r W_{i} \mathrm{~d} u \mathrm{~d} x^{i} .
$$

Here we introduced a 2-dimensional metric

$$
d s_{\top}^{2}=\Sigma^{2} \mathrm{~d} \rho^{2}+\Sigma^{-2} \rho^{2} \mathrm{~d} \phi^{2}
$$

and $r$-independent 1-form $W=W_{i} \mathrm{~d} x^{i}$,

$$
W=2 \frac{\Sigma, \rho}{\Sigma} \mathrm{d} \rho .
$$

These tensors can be understood as tensors on space spanned by two coordinates $\rho, \phi$. This space can be covered by other suitable spatial coordinates $x^{i}$, and we will use the Latin indices $i, j, \ldots$ to label the corresponding tensor components.

By an appropriate transformation of coordinates 7 , 44], the 2-dimensional metric $d s_{\top}^{2}$ can be transformed into a conformally flat form, which in the standard complex null coordinates $\zeta, \bar{\zeta}$ reads

$$
d s_{\top}^{2}=\frac{2}{P^{2}} \mathrm{~d} \zeta \mathrm{d} \bar{\zeta} .
$$

Such a transformation brings the metric (2.13) into the Kundt form.

The gyraton generalization of (2.13) then reads

$$
\begin{aligned}
d s^{2}= & -2 \Sigma^{2} H \mathrm{~d} u^{2}-2 \mathrm{~d} u \mathrm{~d} r+d s_{\perp}^{2} \\
& +2\left(r W_{i}-\Sigma^{2} a_{i}\right) \mathrm{d} u \mathrm{~d} x^{i} .
\end{aligned}
$$

We have added the term $-2 \Sigma^{2} H \mathrm{~d} u^{2}$ which represents a gravitational wave on the Melvin universe [43, 44] with an arbitrary profile function $H$, and the non-diagonal terms $-2 \Sigma^{2} a_{i} \mathrm{~d} u \mathrm{~d} x^{i}$ characteristic for gyratons. It will be shown in the following that these terms can be generated by specific gyratonic matter.

Transforming back to the Melvin coordinate $v$ and cylindrical coordinates $\rho, \phi$, we obtain the ansatz for the metric describing the gyraton on Melvin spacetime, ${ }^{3}$

$$
\begin{aligned}
d s^{2}= & -2 \Sigma^{2} H \mathrm{~d} u^{2}-2 \Sigma^{2} \mathrm{~d} u \mathrm{~d} v+\left(\Sigma^{2} \mathrm{~d} \rho^{2}+\Sigma^{-2} \rho^{2} \mathrm{~d} \phi^{2}\right) \\
& +2 \Sigma^{2}\left(a_{\rho} \mathrm{d} u \mathrm{~d} \rho+a_{\phi} \mathrm{d} u \mathrm{~d} \phi\right) .
\end{aligned}
$$

The function $H(u, v, \rho, \phi)$ can depend on all coordinates, but we assume that the functions $a_{i}(u, \rho, \phi)$ are $v$ independent (it actually follows from the Maxwell equations as will be shown below). Let us note that the previously cited works assumed also the function $H$ $v$ independent.

\footnotetext{
${ }^{3}$ Here we use notation different from [43, 44], we use $-2 H \mathrm{~d} u^{2}$ instead of $-H \mathrm{~d} u^{2}$ to match our notation in 33].
} 


\section{The ansatz for the matter}

The metric should satisfy the Einstein equations with a stress-energy tensor generated by the electromagnetic field and the gyratonic source,

$$
G_{\mu \nu}=\varkappa\left(T_{\mu \nu}^{\mathrm{EM}}+T_{\mu \nu}^{\mathrm{gyr}}\right) .
$$

We assume that the electromagnetic field (2.7) modified by the gyraton is given by

$$
F=E \mathrm{~d} v \wedge \mathrm{d} u+B \Sigma^{-2} \rho \mathrm{d} \rho \wedge \mathrm{d} \phi+\sigma_{j} \mathrm{~d} u \wedge \mathrm{d} x^{j} .
$$

Similar to [33], we have added the term $\sigma_{j} \mathrm{~d} u \wedge \mathrm{d} x^{j}$.

To evaluate Maxwell equations, it is useful to write down the self-dual form of the Maxwell tensor $\mathcal{F}$. The Hodge dual of (2.20) reads

$$
\begin{aligned}
\star F=B \mathrm{~d} v \wedge & \mathrm{d} u-E \Sigma^{-2} \rho \mathrm{d} \rho \wedge \mathrm{d} \phi \\
& +(* \sigma+B a-E * a)_{j} \mathrm{~d} u \wedge \mathrm{d} x^{j},
\end{aligned}
$$

where the $\operatorname{star} *$ means the 2 -dimensional Hodge dual defined on the transversal space, see the next section (IID). For the self-dual Maxwell tensor $\mathcal{F}$ we thus obtain

$$
\begin{aligned}
\mathcal{F}=\mathcal{B}(\mathrm{d} v \wedge \mathrm{d} u & -i \Sigma^{-2} \rho \mathrm{d} \rho \wedge \mathrm{d} \phi \\
& \left.+(\mathcal{S}-i * a)_{j} \mathrm{~d} u \wedge \mathrm{d} x^{j}\right) .
\end{aligned}
$$

where we introduced a complex transverse 1 -form $\mathcal{S}_{j}$,

$$
\mathcal{B S}_{j}=(\sigma+i * \sigma)_{j}+i B(a+i * a)_{j} .
$$

This form is self-dual with respect to the Hodge duality * on the transversal space,

$$
* \mathcal{S}_{j}=-i \mathcal{S}_{j}
$$

and therefore it can be written using a real 1-form $s_{j}$ :

$$
\mathcal{S}_{j}=(s+i * s)_{j}
$$

The original 1-form $\sigma_{i}$ can be expressed in terms of $s_{j}$ as

$$
\sigma_{j}=E s_{j}-B *(s-a)_{j}
$$

In the following we use $s_{j}(v, u, \rho, \phi)$ as a basic variable for the electromagnetic field.

The stress-energy tensor $T_{\mu \nu}^{\mathrm{EM}}$ corresponding to the field (2.22) is given in (A3).

Finally, we must define the gyratonic matter by specifying the structure of its stress-energy tensor. It is obtained from the standard stress-energy tensor of a null fluid by adding terms corresponding to 'internal spatial rotation' of the fluid:

$$
\varkappa T^{\mathrm{gyr}}=j_{u} \mathrm{~d} u^{2}+2 j_{\rho} \mathrm{d} u \mathrm{~d} \rho+2 j_{\phi} \mathrm{d} u \mathrm{~d} \phi .
$$

We admit a general coordinate dependence of the source functions $j_{u}(v, u, \rho, \phi)$ and $j_{j}(v, u, \rho, \phi)$. However, it will be shown below that the field equations enforce a trivial $v$ dependence.

The gyraton source is described only on a phenomenological level, by its stress-energy tensor (2.27), which is assumed to be given, and our aim is to determine its influence on the metric and the electromagnetic field. However, we have to consider that the gyraton stress-energy tensor is locally conserved. It means that the functions $j_{u}$ and $j_{i}$ must satisfy the constraint given by

$$
T_{\mu \nu}^{\mathrm{gyr}} ; \nu=0 .
$$

Of course, if we had considered a specific internal structure of the gyratonic matter, the local energy-momentum conservation would have been a consequence of field equations for the gyraton. Without that, we have to require (2.28) explicitly.

To conclude, the fields are characterized by functions $\Sigma, H, a_{j}$, and $s_{j}$, which must be determined by the field equations provided the gyraton sources $j_{u}$ and $j_{j}$ and the constants $E$ and $B$ are given.

\section{The geometry of the transverse space}

The geometry (2.18) identifies the null geodesic congruence generated by $\partial_{v}$ which is parametrized by an affine parameter $v$, the family of null hypersurfaces $u=$ constant, and 2-dimensional transverse spaces $u, v=$ constant.

The gravitational wave moves along the null direction $\partial_{v}$, i.e., it propagates with the speed of light along the $z$-direction, which is the direction of the electromagnetic field. The hypersurface $u=$ constant corresponds to the surface of the constant 'phase', and the transverse spaces $u, v=$ constant are spatial wave fronts of the wave.

Physical quantities do not depend on the affine parameter $v$, or this dependence is trivial and it will be explicitly found. Specifically, the geometry of the transverse space is $v$ independent.

It turns out to be convenient to restrict various quantities to the transverse space. For example, we can interpret $a_{i}$ and $s_{i}$ as components of $u$-dependent 1 -forms on the transverse space. Our goal is to formulate all equations for physical quantities on the transverse spaces. For that we need to review some properties of the transverse geometry. It was studied in detail in [44], and on a general level in [33], nevertheless it will be useful to mention some of the properties explicitly.

The transverse metric is obtained by restriction of the full metric (2.18) to the transverse space and it is given by the expression (2.14),

$$
d s_{\top}^{2}=g_{\top i j} \mathrm{~d} x^{i} \mathrm{~d} x^{j}=\Sigma^{2} \mathrm{~d} \rho^{2}+\Sigma^{-2} \rho^{2} \mathrm{~d} \phi^{2}
$$

The associated Gauss curvature is given by the scalar curvature $R_{\top}$,

$$
K=\frac{1}{2} R_{\top}=\frac{\varrho_{\mathrm{EM}}}{\Sigma^{4}}\left(2-\frac{1}{4} \varrho_{\mathrm{EM}} \rho^{2}\right) .
$$


It is obvious that only the electromagnetic field is responsible for the non-flatness of the transversal space - it is insensitive to the presence of the gyraton. For $\varrho_{\mathrm{EM}}=0$ the curvature vanishes and we get the flat plane.

In general, the curvature is not constant and it is finite everywhere. The Gauss curvature $K$ has maximum on the axis $\rho=0$ where it is equal to $2 \varrho_{\mathrm{EM}}$; it is positive for $0 \leq \rho<2 \sqrt{2} / \sqrt{\varrho_{\mathrm{EM}}}$, and vanishes on the circle at $\rho=2 \sqrt{2} / \sqrt{\varrho_{\mathrm{EM}}}$. For $\rho>2 \sqrt{2} / \sqrt{\varrho_{\mathrm{EM}}}$, it goes to negative values and it has its minimum $K=-\frac{\varrho_{\mathrm{EM}}}{256}$ at $\rho=2 \sqrt{3} / \sqrt{\varrho_{\mathrm{EM}}}$. Then it grows again, and as $\rho \rightarrow+\infty$ the curvature vanishes, $K \rightarrow 0^{-}$.

The circumference of a circle of constant radius $\rho$ is vanishing when $\rho \rightarrow+\infty$. Therefore, we measure a much shorter circumference for larger $\rho$-as if we would move "along the stem of the wine-glass toward the narrowing end," [40, 41, 44].

The 2-dimensional Levi-Civita tensor $\epsilon_{i j}$ associated with the metric (2.29) is $\epsilon=\rho \mathrm{d} \rho \wedge \mathrm{d} \phi$. The covariant derivative will be denoted by a colon, e.g., $a_{i: j}$. We raise and lower the Latin indices using $g_{\top i j}$, which differs from lowering indices using $g_{\alpha \beta}$ thanks to non-vanishing terms $g_{u i}$. We use a shorthand for a transverse square of the norm of a 1-form $a_{i}$ as

$$
a^{2} \equiv a^{i} a_{i}=\Sigma^{-2} a_{\rho}^{2}+\rho^{-2} \Sigma^{2} a_{\phi}^{2} .
$$

In two dimensions, the Hodge duals of 0,1 and 2-forms $\varphi, a_{i}$, and $f_{i j}$, respectively, read

$$
(* \varphi)_{i j}=\varphi \epsilon_{i j}, \quad(* a)_{i}=a_{j} \epsilon^{j}{ }_{i}, \quad * f=\frac{1}{2} f_{i j} \epsilon^{i j}=\frac{1}{\rho} f_{\rho \phi} .
$$

For convenience, we also introduce an explicit notation for 2-dimensional divergence and rotation of a transverse 1 -form $a_{i}$

$$
\begin{aligned}
& \operatorname{div} a \equiv a_{i}: i=\frac{1}{\Sigma^{2}} a_{\rho, \rho}+\frac{\Sigma^{2}}{\rho^{2}} a_{\phi, \phi}+\frac{1}{\rho \Sigma^{2}} a_{\rho}-\frac{\Sigma_{, \rho}^{2}}{\Sigma^{4}} a_{\rho}, \\
& \operatorname{rot} a \equiv \epsilon^{i j} a_{j, i}=\frac{1}{\rho}\left(a_{\phi, \rho}-a_{\rho, \phi}\right) .
\end{aligned}
$$

For 2 -form $f_{i j}$ we get

$$
\operatorname{div} f \equiv f_{i j}: j=\frac{1}{\Sigma^{2}}\left(f_{\phi \rho, \rho}-\frac{1}{\rho} f_{\phi \rho}\right) \mathrm{d} \phi+\frac{\Sigma^{2}}{\rho^{2}} f_{\rho \phi, \phi} \mathrm{d} \rho,
$$

and $\operatorname{rot} f=0$. We can generalize the action of divergence and rotation also on a scalar function $f$ as $\operatorname{div} f=0$ and $\operatorname{rot} f=-* \mathrm{~d} f$. Note that the divergence and rotation are related as $\operatorname{div} a=\operatorname{rot} * a$, and the relation to the transverse exterior derivative is $\mathrm{d} a=* \operatorname{rot} a$. Clearly, $\operatorname{div} \operatorname{div} a=0, \operatorname{div} \operatorname{rot} a=0$, and $\operatorname{rot} \mathrm{d} a=0$.

The Laplace operator of a function $\psi$ reads

$$
\Delta \psi=\psi_{: i}: i=\frac{1}{\Sigma^{2}} \psi_{, \rho \rho}+\frac{\Sigma^{2}}{\rho^{2}} \psi_{, \phi \phi}+\frac{1}{\rho \Sigma^{2}} \psi_{, \rho}-\frac{\Sigma_{, \rho}^{2}}{\Sigma^{4}} \psi_{, \rho},
$$

and for a transverse 1-form $\eta$ it is defined as $\triangle \eta \equiv \mathrm{d} \operatorname{div} \eta-\operatorname{rot} \operatorname{rot} \eta$.
Finally, the transverse space is topologically trivial since it has topology of a plane. We can thus assume that the Poincare lemma $(\mathrm{d} \omega=0 \Rightarrow \omega=\mathrm{d} \sigma)$ holds, which in terms of rotation and divergence means that $\operatorname{div} \omega=0$ implies $\omega=\operatorname{rot} \sigma$. However, since the transverse space is non-compact and we do not know a priori boundary conditions for various quantities at infinity $\rho \rightarrow \infty$, we have to admit non-trivial harmonics. Therefore, we cannot assume a uniqueness of the Hodge decomposition. Moreover, in some cases it can be physically relevant to consider also topologically nontrivial harmonics which are singular, e.g., at the origin $\rho=0$. Such solutions would correspond to fields around singular sources localized on the axis. However, we will ignore these cases in a general discussion.

\section{THE FIELD EQUATIONS}

\section{A. The field equations for matter}

Now, we will investigate the equations for matter, i.e., the Maxwell equations for electromagnetic field and the condition (2.28) for the gyraton source.

Both Maxwell equations for real Maxwell tensor are equivalent to the cyclic Maxwell equation for the selfdual Maxwell tensor (2.22),

$$
\begin{aligned}
\mathrm{d} \mathcal{F}=\mathcal{B}[ & \partial_{v}(s+i *(s-a))_{j} \mathrm{~d} v \wedge \mathrm{d} u \wedge \mathrm{d} x^{j} \\
& -(\operatorname{rot} s+i \operatorname{div}(s-a)) \mathrm{d} u \wedge \epsilon]=0 .
\end{aligned}
$$

From the real part we immediately get that the 1 -form $s_{i}$ is $v$ independent, $\partial_{v} s_{i}=0$, and rotation-free,

$$
\operatorname{rot} s=0 \text {. }
$$

From the imaginary part it follows that the 1 -form $a_{i}$ is also $v$ independent (as we have already mentioned above) and it satisfies

$$
\operatorname{div}(s-a)=0 .
$$

Equations (3.2) and (3.3) guarantee the existence and determine the structure of potentials which will be discussed in detail in Section IIIC.

Next, we analyze the condition (2.28) for the gyraton source. When translated to the transverse space, it gives

$$
-\partial_{v} j_{i} \mathrm{~d} x^{i}+\left(-\partial_{v} j_{u}+\operatorname{div}\left(\Sigma^{2} j\right)+\Sigma^{2} a^{i} \partial_{v} j_{i}\right) \mathrm{d} u=0 .
$$

The source functions $j_{i}$ must be thus $v$ independent and $j_{u}$ has to have the structure

$$
j_{u}=v \operatorname{div}\left(\Sigma^{2} j\right)+\iota
$$

where $\iota\left(u, x^{i}\right)$ is a $v$ independent function. The gyraton source (2.27) is therefore fully determined by three $v$-independent functions $\iota\left(u, x^{j}\right)$ and $j_{i}\left(u, x^{j}\right)$. 
Equation (3.5) gives us also an insight into interpretation of the gyratonic terms $j_{i}$. They are composed from two contributions: one is related to a kind of 'heat flow' which changes energy $j_{u}$ of the fluid, and the other which is related to intrinsic rotation of the fluid. The source representing 'heat flow' has thus non-vanishing divergence $\operatorname{div}\left(\Sigma^{2} j\right)$ and we require a vanishing rotational part $\operatorname{rot}\left(\Sigma^{2} j\right)$. In opposite, the source representing intrinsic rotation has vanishing heat flow, i.e., it satisfies

$$
\operatorname{div}\left(\Sigma^{2} j\right)=0 .
$$

Such a source can be written in terms of a rotational potential $\nu$ as $j=-\Sigma^{-2} \operatorname{rot} \nu$. In components it means

$$
j_{\rho}=-\frac{1}{\rho} \nu_{,}, \quad j_{\phi}=\frac{\rho}{\Sigma^{4}} \nu_{, \rho} .
$$

Physically more relevant is the rotational part of the source, since it can describe the spin of the null fluid, or, in a specific limit, of the polarized beam of light. Terms related to heating flow have bad causal behavior and therefore typically do not satisfy various energy conditions and they are thus rather unphysical. Interpretation of the gyraton source was discussed previously also in 21, 22, 26, 33].

\section{B. The Einstein equations}

The Einstein gravitational law (2.19) needs the Einstein tensor and the electromagnetic and gyraton stressenergy tensors. These quantities can be found in the Appendix $\mathrm{A}$. We can combine them and inspect various components of the Einstein equations.

The $v u$-component determines the function $\Sigma$, namely it gives the condition

$$
-\rho\left(\Sigma_{, \rho}\right)^{2}+2 \Sigma \Sigma_{, \rho}=\varrho_{\mathrm{EM}} \rho .
$$

It is straightforward to check that it is satisfied again by $\Sigma$ in the Melvin form (2.3).

The transverse diagonal components $\rho \rho$ and $\phi \phi$ require

$$
\partial_{v}^{2} H=0
$$

thus we obtain the explicit $v$ dependence of the metric function $H$ as

$$
H=g v+h,
$$

where we have introduced $v$-independent functions $g\left(u, x^{j}\right)$ and $h\left(u, x^{j}\right)$.

The remaining nontrivial components of the Einstein equations are those involving the gyraton source (2.27). The $u i$-components give the equation related to $j_{i}$,

$$
j_{i}=\frac{1}{2} \Sigma^{2} f_{i j}^{i j}-\left(\Sigma^{2}\right)_{, k} g^{k j} f_{j i}+g_{, i}+\frac{2 \varrho_{\mathrm{EM}}}{\Sigma^{2}}\left(s_{i}-a_{i}\right),
$$

where we introduce the exterior derivative $f_{i j}$ of the transverse 1-form $a_{i}$ and its Hodge dual $b\left(u, x^{j}\right)$,

$$
\begin{gathered}
f_{i j} \equiv a_{j, i}-a_{i, j}=(* b)_{i j}, \\
b \equiv * f=\operatorname{rot} a .
\end{gathered}
$$

In terms of $b$, equation (3.11) can be rewritten as

$$
\Sigma^{2} j=\frac{1}{2} \operatorname{rot}\left(\Sigma^{4} b\right)+\Sigma^{2} \mathrm{~d} g+2 \varrho_{\mathrm{EM}}(s-a) .
$$

Here and in the following $\mathrm{d} g$ represents just the transverse gradient $\mathrm{d} g=g_{, i} \mathrm{~d} x^{i}$, and for simplicity we skipped the transverse indices.

It is useful to split the equation into divergence and rotation parts by applying div and rot:

$$
\begin{aligned}
& \operatorname{div}\left(\Sigma^{2} j\right)=\operatorname{div}\left(\Sigma^{2} \mathrm{~d} g\right), \\
& \operatorname{rot}\left(\Sigma^{2} j\right)=-\frac{1}{2} \triangle\left(\Sigma^{4} b\right)+\operatorname{rot}\left(\Sigma^{2} \mathrm{~d} g\right)-2 \varrho_{\mathrm{EM}} b .
\end{aligned}
$$

Here we have used the relations (3.2) and (3.13). The formula (3.15) is the equation for $g$, (3.16) is the equation for $b$ and together with (3.13) it determines $a_{i}$. In the next section we will return to these equations introducing suitable potentials which allow us to escape the necessity of taking an additional derivative of (3.14) when deriving the equation for $a_{i}$.

Finally, from the $u u$-component of the Einstein equation we obtain

$$
\begin{aligned}
j_{u}= & \operatorname{div}\left(\Sigma^{2} \mathrm{~d} g\right) v+\Sigma^{2}\left(\Delta h-\left(\Sigma^{-2}\right)_{, \rho} h_{, \rho}\right) \\
& +\frac{1}{2} \Sigma^{4} b^{2}+2 \Sigma^{2} a^{i} g_{, i}+\partial_{u} \operatorname{div}\left(\Sigma^{2} a\right)+g \operatorname{div}\left(\Sigma^{2} a\right) \\
& -2 \varrho_{\text {EM }}(s-a)^{2} .
\end{aligned}
$$

When we compare the coefficient in front of $v$ with (3.15) we find that it has structure consistent with (3.5). The nontrivial $v$-independent part of (3.17) gives the equation for the metric function $h$,

$$
\begin{aligned}
& \Sigma^{2}\left(\Delta h-\left(\Sigma^{-2}\right)_{, \rho} h_{, \rho}\right)=\iota-\frac{1}{2} \Sigma^{4} b^{2}-2 \Sigma^{2} a^{i} g_{, i} \\
& \quad-\partial_{u} \operatorname{div}\left(\Sigma^{2} a\right)-g \operatorname{div}\left(\Sigma^{2} a\right)+2 \varrho_{\mathrm{EM}}(s-a)^{2} .
\end{aligned}
$$

\section{Introducing potentials}

In the previous section we have found that the Maxwell and Einstein equations reduce to two potential equations (3.2), (3.3), and two source equations (3.11), (3.17).

According to the two dimensional Hodge decomposition we can express the 1 -form $a_{i}$ using two scalar potentials $\kappa\left(u, x^{j}\right)$ and $\lambda\left(u, x^{j}\right)$,

$$
a=\mathrm{d} \kappa+\operatorname{rot} \lambda .
$$

These potentials control the divergence and the rotation of $a_{i}$ as

$$
\operatorname{div} a=\Delta \kappa, \quad \operatorname{rot} a=-\Delta \lambda .
$$


Comparing with (3.13) we thus obtain the equation for $\lambda$ in terms of $b$,

$$
\Delta \lambda=-b
$$

The first potential equation (3.2) gives immediately that $s_{i}$ has a potential $\varphi\left(u, x^{j}\right)$,

$$
s=\mathrm{d} \varphi .
$$

Equation (3.3) implies that there exists a potential $\psi\left(u, x^{j}\right)$ satisfying

$$
s-a=-\operatorname{rot} \psi
$$

In terms of these potentials the 1-form (2.26) from the real Maxwell tensor (2.7) reads

$$
\sigma=E \mathrm{~d} \varphi+B \mathrm{~d} \psi
$$

The potential $\varphi$ and $\psi$ are not, however, independent. Substituting (3.19) and (3.22) into (3.23) we obtain the key relation among the potentials:

$$
\mathrm{d}(\varphi-\kappa)+\operatorname{rot}(\psi-\lambda)=0
$$

If the Hodge decomposition was unique, the gradient and rotational parts would be vanishing separately, i.e., we would get $\varphi=\kappa$ and $\psi=\lambda$ (up to unimportant constants). The non-uniqueness of the Hodge decomposition is linked to the possible existence of a non-trivial harmonic 1-form $\eta_{i}\left(u, x^{j}\right)$,

$$
\triangle \eta=0
$$

in terms of which the gradient and rotation parts of (3.25) can be expressed as

$$
\begin{aligned}
& \varphi-\kappa=\operatorname{div} \eta \\
& \psi-\lambda=-\operatorname{rot} \eta
\end{aligned}
$$

These are equations for electromagnetic potentials $\varphi$ (or $\psi$, respectively) in terms of the metric potentials $\kappa$ and $\lambda$. The 1-form $\eta$ encodes an extra freedom, which allows a nontrivial electromagnetic field not uniquely determined by the metric. (Such contributions would allow one to take into account, for example, an additional electromagnetic charge localized at the origin of the transverse space, cf. the discussion of particular cases in section [V]) However, sufficiently restrictive conditions at the infinity and the smoothness on the whole transverse space for the potentials would eliminate this freedom, so the case $\eta=0$ is a rather representative choice.

After eliminating the electromagnetic potentials, we need to formulate the equations for $\kappa$ and $\lambda$. We start with the divergence part of (3.14) which can be rewritten using the modified Laplace operator acting on the metric function $g$ :

$$
\Sigma^{2}\left(\Delta g-\left(\Sigma^{-2}\right)_{, \rho} g_{, \rho}\right)=\operatorname{div}\left(\Sigma^{2} j\right) .
$$

However, for $g$ solving this equation, (3.15) is also the integrability condition for the quantity $\Sigma^{2}(\mathrm{~d} g-j)$. It can thus be written in terms of a potential $\omega$,

$$
\Sigma^{2}(\mathrm{~d} g-j)=\operatorname{rot} \omega
$$

or, more explicitly,

$$
\mathrm{d} \omega=-\Sigma^{2}(\operatorname{rot} g+* j) .
$$

For the source (3.7) without intrinsic 'heating', the right-hand side of (3.29) is zero and the function $\omega$ has an additive contribution from the rotational potential $\nu$, namely it has to satisfy

$$
\mathrm{d}(\omega-\nu)=-\Sigma^{2} \operatorname{rot} g
$$

The function $\omega$ contains information from the source $j$ and from the metric function $g$ relevant for the rotational part of equation (3.14). Indeed, substituting the potentials and rot $\omega$ into (3.14) we obtain

$$
\operatorname{rot}\left(\frac{1}{2} \Sigma^{4} b-2 \varrho_{\mathrm{EM}} \psi+\omega\right)=0 \text {. }
$$

Substituting (3.21), (3.28), and integrating (absorbing an integration constant to $\omega$ ), we derive the equation for the potential $\lambda$

$$
\frac{1}{2} \Sigma^{4} \Delta \lambda+2 \varrho_{\mathrm{EM}} \lambda=\omega+2 \varrho_{\mathrm{EM}} \operatorname{rot} \eta
$$

Taking into account relations (3.28) and (3.26), we obtain the alternative equation for $\psi$

$$
\frac{1}{2} \Sigma^{4} \Delta \psi+2 \varrho_{\mathrm{EM}} \psi=\omega
$$

The other metric potential $\kappa$ remains unrestricted. This non-uniqueness is related to the gauge freedom discussed in detail in section IIIE. This coordinate freedom allows us to set the potential $\kappa$ to an arbitrary convenient form, e.g., to eliminate it completely.

\section{Discussion of the field equations}

We have thus formulated all field equations as equations on the transverse space. They are written in a separated form, i.e., they can be solved one after the other: First, one has to find harmonic 1-form $\eta$ satisfying equation (3.26) and metric function $g$ satisfying (3.15). It allows one to integrate the function $\omega$ which together with $\operatorname{rot} \eta$ appears as a source in equation (3.34) for the potential $\lambda$. Using the gauge freedom one can choose the other potential $\kappa$. Equations (3.27) and (3.28) determine electromagnetic potentials and through equation (2.26) the electromagnetic field. Finally, the remaining metric function $h$ is determined by equation (3.18), in which the previously computed quantities contribute to the source on the right-hand side. 
The most complicated field equations-3.29), (3.18), and (3.34) - are partial differential equations on the 2-dimensional space, which are solvable, at least in principle. They all contain a modified Laplace operator, equations (3.29) and (3.18) for $g$ and $h$ the same one, namely

$$
\Sigma^{2}\left(\Delta f-\left(\Sigma^{-2}\right)_{, \rho} f_{, \rho}\right) \equiv \operatorname{div}\left(\Sigma^{2} \mathrm{~d} f\right) .
$$

Solutions for particular cases (assuming rotational symmetry) will be discussed in section IV.

It is important to observe that, except equation (3.18) for $h$, the field equations are linear. We can thus superpose two solutions simply by adding the fields together. Only in the last step, when computing the source for equation (3.18), one has to include total superposition of the fields $g, a_{i}$, and $\sigma_{i}$ since the expression for the source is non-linear.

Finally, we have not paid much attention to the $u$ dependence of the studied quantities. All metric functions, matter fields and sources can depend on the coordinate $u$ and this dependence does not enter the field equations except in one term on the right-hand side of equation (3.18). The profile of the gyraton in the $u$ direction can thus be specified arbitrarily. It corresponds to the fact that both matter and gravitational field move with the speed of light and information on different hypersurfaces $u=$ constant evolves rather independently.

Also the dependence of the metric and fields on the coordinate $v$ is very simple and it was found for all quantities explicitly.

\section{E. The gauge transformation}

The coordinate transformation $\tilde{v} \rightarrow v=\tilde{v}-\chi\left(u, x^{j}\right)$ accompanied by the following redefinition of the metric functions and matter fields:

$$
\begin{gathered}
v=\tilde{v}-\chi, \\
g=\tilde{g}, \quad h=\tilde{h}+\tilde{g} \chi+\partial_{u} \chi, \quad a_{i}=\tilde{a}_{i}-\chi, i \\
s_{i}=\tilde{s}_{i}-\chi, i, \quad \sigma_{i}=\tilde{\sigma}_{i}-E \chi_{, i}, \\
\kappa=\tilde{\kappa}-\chi, \quad \lambda=\tilde{\lambda}, \quad \varphi=\tilde{\varphi}-\chi, \quad \psi=\tilde{\psi}, \\
\quad \omega=\tilde{\omega}, \quad \eta=\tilde{\eta}, \\
j_{i}=\tilde{j}_{i}, \quad \iota=\tilde{\iota}+\chi \operatorname{div} j,
\end{gathered}
$$

leaves the metric, the Maxwell tensor, and the gyraton stress-energy tensor in the same form. Therefore, all the field equations remain the same. This transformation is thus a pure gauge transformation and we can use it to simplify the solution of the equations.

There are two natural choices of gauge: we can eliminate either the metric potential $\kappa$ or the electromagnetic potential $\varphi$. In the first case $a=\operatorname{rot} \lambda$ and $\varphi=\operatorname{div} \eta$. In the latter case $s=0, \sigma=B \mathrm{~d} \psi, \kappa=-\operatorname{div} \eta$, and $a=\operatorname{rot} \psi$.

Let us mention that in 33] an analogous gauge transformation allowed us to choose also the metric function g. For the gyraton on the Melvin universe the metric function $g$ decouples from $\kappa$ and it is gauge independent.

The discussed gauge transformation has a clear geometrical meaning: it corresponds to a shift of the origin of the affine parameter $v$ of the null congruence $\partial_{v}$. Note that such a change redefines transverse spaces.

\section{SPECIAL CASES}

In this section we will study the special solutions of the field equations. Namely, we restrict to the axially symmetric situation, i.e., to the case when the geometry and the fields are invariant under action generated by the rotational vector $\partial_{\phi}$. Further, we concentrate on the gyraton generated by a thin beam of matter concentrated at the origin of the transverse space which means on the axis of symmetry.

Thank to linearity mentioned at the end of section IIID, we can discuss various special cases separately. However, the geometry of the spacetime with gyraton is not merely a superposition of the individual contribution since the nonlinear coupling in the metric function $h$.

We do not discuss in detail the $u$ dependence of the fields. It does not enter the field equations, except in the source term of equation (3.18) for $h$. The $u$ dependence of the gyraton sources and corresponding dependence of other fields can thus be chosen arbitrarily.

The symmetry assumption enforces that quantities $a_{i}$, $g, h, \sigma_{i}, j_{i}$, and $\iota$ are $\phi$-independent. It can induce a slightly weaker condition on the potentials: typically, they have a linear dependence on $\phi$.

The thin beam approximation requires that gyratonic matter is concentrated at the origin $o$ of the transverse space given by $\rho=0$, i.e., $j_{i}$ and $\iota$ should be distributions with the support at the origin. However, we relax this condition slightly in the case of gyratonic 'heat flow' discussed in section IVB.

In all discussed cases we use a natural gauge

$$
\kappa=0, \text { i.e., } \quad a=\operatorname{rot} \lambda \text {. }
$$

Together with the symmetry assumptions it implies $\lambda_{, \phi}=$ constant.

\section{A. Pure gravitational gyraton}

We start with the simplest vacuum case: we set $j_{i}=0$ and $\iota=0$ and we assume no pure electromagnetic contribution, i.e., $\eta=0$. The equation for $g$ has only a trivial solution $g=g_{\mathrm{o}}=$ constant, the function $\omega$ is also a trivial constant and we obtain equation (3.34) with vanishing right-hand side. Taking into account that $\lambda_{, \phi}=$ constant we obtain that $\lambda$ must be $\phi$ independent (a $\phi$-linear term in $\lambda$ would require an analogous term in the source) and 
we obtain an ordinary differential equation

$$
\frac{1}{\rho}\left(\frac{\rho}{\Sigma^{2}} \lambda_{, \rho}\right)_{, \rho}+\frac{4 \varrho_{\mathrm{EM}}}{\Sigma^{4}} \lambda=0 .
$$

Substituting (2.3) for $\Sigma$, it is possible to obtain two independent solutions, one regular at the origin,

$$
\lambda=-\gamma \Sigma^{-1}\left(1-\frac{3}{4} \varrho_{\mathrm{EM}} \rho^{2}\right),
$$

and the other behaving as $\log \rho$ near the origin, which corresponds to a delta source at the origin and it will be discussed in section IVD,

The metric 1-form $a=\operatorname{rot} \lambda$ has thus components

$$
a_{\rho}=0, \quad a_{\phi}=-\frac{\rho}{\Sigma^{2}} \lambda_{, \rho}=-\gamma \frac{2 \varrho_{\mathrm{EM}}}{\Sigma^{4}} \rho^{2},
$$

and its 'strength' $b=\operatorname{rot} a$ is then

$$
b=-\gamma \frac{4 \varrho_{\mathrm{EM}}}{\Sigma^{5}}\left(1-\frac{3}{4} \varrho_{\mathrm{EM}} \rho^{2}\right) .
$$

These can be plugged into (3.18) which turns out to be

$$
\frac{1}{\rho}\left(\rho h_{, \rho}\right)_{, \rho}=-\gamma^{2} \frac{8 \varrho_{\mathrm{EM}}^{2}}{\Sigma^{6}}\left(1-\frac{1}{4} \varrho_{\mathrm{EM}} \rho^{2}\right)\left(1-\frac{9}{4} \varrho_{\mathrm{EM}} \rho^{2}\right) .
$$

It can be integrated explicitly; however, the result is rather long, so we skip it.

\section{B. Non-spinning beam with 'heat' flow}

As we discussed in section $\amalg$ IIA the gyraton source can have two contributions: one, which changes gyratonic energy density $j_{u}$ and the other corresponding to intrinsic rotation. Let us investigate the case when the gyratonic energy is concentrated at the origin (a thin beam) but there is axially symmetric energy flow in the $\partial_{\rho}$ direction which accumulates energy at the beam (a 'heating' process). Namely, we assume that $\partial_{v} j_{u}=\operatorname{div}\left(\Sigma^{2} j\right)$ is nonzero only at the origin, so elsewhere the transverse flow $j_{i}$ must satisfy equation (3.6). Such $j_{i}$ has the form

$$
j_{\rho}=\frac{\alpha}{2 \pi} \frac{1}{\rho}, \quad j_{\phi}=0,
$$

and the increasing energy of the gyraton is given by

$$
j_{u}=\alpha v \delta_{o} .
$$

Here, $\delta_{o}$ stands for the transverse delta function localized at the origin $o$, normalized to the standard metric volume element on the transverse space.

Since the 'heating' is localized only at the origin, the gyraton source (4.7) can be locally written using the source potential $\nu$, cf. (3.7), as

$$
\nu=-\frac{\alpha}{2 \pi} \phi
$$

Note however, that the potential cannot be defined globally and it is not well behaved at the origin.

We again assume no pure electromagnetic contribution, i.e., $\eta=0$. The requirement of the axial symmetry enforces that the difference $\Sigma^{-2} \operatorname{rot} \omega$ between $\mathrm{d} g$ and $j$, cf. (3.30), must be zero, i.e., $\omega=0$. The $\phi$-independent metric function $g$ must thus satisfy $g_{, \rho}=j_{\rho}$ which gives

$$
g=\frac{\alpha}{2 \pi} \log \rho+g_{\mathrm{o}} .
$$

The equation for $\lambda$ has again the form (4.2) and in this case we choose the trivial solution $\lambda=0$. With the gauge $\kappa=0$ we thus obtain the only nontrivial field to be the metric function $g$, otherwise $a_{i}=0$ and $\sigma_{i}=0$. The source for equation (3.18) is also trivial, given only by $\iota$, and we will study it in the next case.

\section{Non-spinning light beam}

A particular example of the gyraton source is standard null fluid. The thin non-spinning beam localized at the origin is described by the source

$$
\iota=\varepsilon \delta_{o}, \quad j_{i}=0 .
$$

We can set all the fields except $h$ to be zero: $g=0$, $a_{i}=0$, and $\sigma_{i}=0$. The equation for $h$ outside the origin is $\rho^{-1}(\rho h, \rho), \rho=0$ which (with proper fixing of the source constant) gives

$$
h=\frac{\varepsilon}{2 \pi} \log \rho .
$$

\section{Thin gyraton-spinning light beam}

Finally we proceed with the most characteristic representant of the gyratonic matter. It is a simple null beam of energy localized at the origin with no heating, which, however, contains an intrinsic energy rotation. Since we have a point-like source at the transverse space, we can speak about inner spin instead of a global rotational energy flow.

The gyraton source has a form ${ }^{4}$

$$
\begin{gathered}
\iota=\varepsilon \delta_{o}, \\
j=-\Sigma^{-2} \operatorname{rot} \nu, \quad \text { with } \quad \nu=\beta \delta_{o} .
\end{gathered}
$$

The symmetry assumptions together with the no-heating requirement implies $g=$ constant, which we choose to be zero in this case. Equation (3.30) also implies that $\omega=\nu$.

\footnotetext{
4 The exact structure of the singular source $j_{i}$ at the origin can be read out from the singular solution (4.16) of equation (3.34) for $\lambda$ below.
} 
Ignoring again the electromagnetic contribution, $\eta=0$, we obtain the equation for $\lambda$

$$
\frac{1}{\rho}\left(\frac{\rho}{\Sigma^{2}} \lambda_{, \rho}\right)_{, \rho}+\frac{4 \varrho_{\mathrm{EM}}}{\Sigma^{4}} \lambda=\Sigma^{-4} \nu .
$$

The solution of the homogeneous equation with a singular behavior $\sim \log \rho$ corresponding to the delta function (4.14) at the origin reads

$$
\begin{aligned}
\lambda=-\frac{\beta}{2 \pi} \Sigma^{-1} & \left(1+\frac{1}{2} \varrho_{\mathrm{EM}} \rho^{2}-\frac{3}{64} \varrho_{\mathrm{EM}}^{2} \rho^{4}-\frac{1}{768} \varrho_{\mathrm{EM}}^{3} \rho^{4}\right. \\
& \left.+\frac{1}{2}\left(1-\frac{3}{4} \varrho_{\mathrm{EM}} \rho^{2}\right) \log \left(\frac{1}{4} \varrho_{\mathrm{EM}} \rho^{2}\right)\right) .
\end{aligned}
$$

The multiplicative constant in the argument of the logarithm can be chosen arbitrary since it generates only an additional homogeneous contribution of the form (4.3).

The metric 1-form $a_{i}$ and its rotation $b$ are

$$
\begin{aligned}
& a_{\rho}=0, \\
& \begin{aligned}
a_{\phi}=-\frac{\beta}{2 \pi} \Sigma^{-4} & \left(1-\frac{3}{8} \varrho_{\mathrm{EM}}^{2} \rho^{4}-\frac{1}{32} \varrho_{\mathrm{EM}}^{3} \rho^{6}-\frac{1}{768} \varrho_{\mathrm{EM}}^{4} \rho^{8}\right. \\
& \left.-\varrho_{\mathrm{EM}} \rho^{2} \log \left(\frac{1}{4} \varrho_{\mathrm{EM}} \rho^{2}\right)\right),
\end{aligned}
\end{aligned}
$$

and

$$
\begin{aligned}
b=2 & \frac{\beta}{2 \pi} \varrho_{\mathrm{EM}} \Sigma^{-5}\left(\left(1-\frac{3}{4} \varrho_{\mathrm{EM}} \rho^{2}\right) \log \left(\frac{1}{4} \varrho_{\mathrm{EM}} \rho^{2}\right)\right. \\
& \left.+2+\varrho_{\mathrm{EM}} \rho^{2}-\frac{3}{32} \varrho_{\mathrm{EM}}^{2} \rho^{4}-\frac{1}{384} \varrho_{\mathrm{EM}}^{3} \rho^{6}\right) .
\end{aligned}
$$

The source for equation (3.18) becomes cumbersome and lengthy, but treatable, in principle.

\section{E. Electromagnetic wave}

In the previous examples we have ignored the possibility of a nontrivial electromagnetic field. Namely, we have assumed that the electromagnetic field is given by the metric potentials via relations $\varphi=\kappa$ and $\psi=\lambda$. However, we have already observed that equation (3.25) admits also other solutions which we have parametrized using a harmonic 1-form $\eta$. To include such solutions we should classify all 1-form harmonics on the transverse space. But if we restrict to the axially symmetric fields we can solve the potential equation (3.25) directly, without referring to $\eta$ explicitly.

Let us study a pure electromagnetic contribution to the matter, i.e., we assume $\iota=0, j_{i}=0$ here. We can thus take a trivial vanishing solution for $g$ which implies $\omega=0$.

The symmetry assumptions tell us that 1-forms $s, \sigma$, and $a$ are $\phi$ independent, which implies that all potentials including $\varphi-\kappa$ and $\psi-\lambda$ can be at most linear in $\phi$ :

$$
\varphi-\kappa=\hat{\varphi}(\rho)+\varphi_{\phi} \phi, \quad \psi-\lambda=\hat{\psi}(\rho)+\psi_{\phi} \phi,
$$

$\varphi_{\phi}, \psi_{\phi}$ being constants and $\hat{\varphi}$ and $\hat{\psi}$ functions of $\rho$ only. Substituting into (3.25) we get

$$
\left(\hat{\varphi}_{, \rho}-\frac{\Sigma^{2}}{\rho} \psi_{\phi}\right) \mathrm{d} \rho+\left(\varphi_{\phi}+\frac{\rho}{\Sigma^{2}} \hat{\psi}_{, \rho}\right) \mathrm{d} \phi=0,
$$

which implies

$$
\hat{\varphi}_{, \rho}=\frac{\Sigma^{2}}{\rho} \psi_{\phi}, \quad \hat{\psi}_{, \rho}=-\frac{\Sigma^{2}}{\rho} \varphi_{\phi} .
$$

Taking into account (2.3), it can be easily integrated and substituting back to (4.19) we obtain

$$
\begin{aligned}
& \varphi=\kappa+\psi_{\phi}\left(\log \rho+\frac{1}{8} \varrho_{\mathrm{EM}} \rho^{2}\right)+\varphi_{\phi} \phi, \\
& \psi=\lambda-\varphi_{\phi}\left(\log \rho+\frac{1}{8} \varrho_{\mathrm{EM}} \rho^{2}\right)+\psi_{\phi} \phi .
\end{aligned}
$$

At this moment it is easier to solve equation (3.35) for $\psi$ with vanishing right-hand side instead of equation (3.34) for $\lambda$. It has solutions in the form (4.3) and (4.16). The metric potential $\lambda$ is then given by the second of the equations (4.19). The metric potential $\kappa$ is vanishing thanks to our gauge.

After choosing the solution for $\psi$, we can thus compute all quantities $a, b, s$, and $\sigma$ and substitute them to equation (3.18) for $h$.

Let us mention that solution (4.19) is singular at the origin. A careful distributional calculation would show that equations for the potentials (3.22) and (3.23) may not be satisfied at the origin. Tracing this singular term back to Maxwell equations, it could lead to non-vanishing electric charges localized at the origin. However, since we have not written down the Maxwell equations with sources, we do not discuss these terms in more detail.

\section{PROPERTIES OF THE GYRATON SPACETIMES}

\section{A. Gravitational field}

In this section we discuss some of the geometrical properties of the gyratonic solutions.

One of the important characteristics of spacetimes are the scalar polynomial invariants which are constructed only from the curvature and its covariant derivatives. It was shown that gyratons in the Minkowski spacetime 22] have all the scalar polynomial invariants vanishing (VSI spacetimes) [23], the gyratons in the anti-de Sitter 26] and direct product spacetimes [33] have all invariants constant (CSI spacetimes) 29].

In these cases, the invariants are independent of all metric functions which characterize the gyraton, and have the same values as the corresponding invariants of the background spacetime. We observe that similar property is valid also for the gyraton on Melvin spacetime, however, in this case the invariants are generally nonconstant, namely, they depend on the coordinate $\rho$. This 
property is a consequence of the general theorem holding for the relevant subclass of the Kundt solution, see Theorem II.7 in [56].

Values of some of the scalar curvature invariants can be found in Appendix C.

The metric (2.18) admits the null vector $k=\partial_{v}$. It is a Killing vector for $g=0$ and $\operatorname{div}\left(\Sigma^{2} j\right)=0$, i.e., for the no 'heating' part in the gyratonic source. The covariant derivative of $k$ is given by

$$
k_{\alpha ; \beta}=-\Sigma^{-2}\left(\partial_{v} H\right) k_{\alpha} k_{\beta}+\Sigma^{-2} k_{[\alpha} \nabla_{\beta]} \Sigma^{2} .
$$

We observe that the congruence is not even recurrent [6] as in the case of a gyraton on direct product spacetimes [33]. For $\partial_{v} H=0$ we recover the formula in Garfinkle and Melvin [43]. In general, the non-recurrency of the congruence is related to the non-vanishing spin coefficient $\tau_{\mathrm{NP}}=-\frac{1}{\sqrt{2}} \frac{1}{\Sigma^{2}} \Sigma_{, \rho}$, cf. [23], calculated in Appendix B]

The null character of $k$ and the condition (5.1) imply that the null congruence with tangent vector $k$ is geodesic, expansion-free, sheer-free and twist-free, and the spacetime thus belongs to the Kundt class.

Next we calculate components of the curvature tensors with respect to the following adapted null tetrad $\{k, l, m, \bar{m}\}[6]$

$$
\begin{aligned}
k & =\partial_{v} \\
l & =\frac{1}{\Sigma^{2}}\left(\partial_{u}-H \partial_{v}\right), \\
m & =\frac{1}{\sqrt{2} \Sigma}\left(a_{m} \partial_{v}+\partial_{\rho}-i \frac{\Sigma^{2}}{\rho} \partial_{\phi}\right) .
\end{aligned}
$$

Here, we have introduced the projection of a transverse 1-form $a$ on the vector $m$

$$
a_{m}=m^{i} a_{i}=a_{\rho}-i \frac{\Sigma^{2}}{\rho} a_{\phi}=(a+i * a)_{\rho},
$$

and we will use an analogous notation also for components of the transverse gradient of a real function $f$

$$
f_{, m}=m^{i} f_{, i}=f_{, \rho}-i \frac{\Sigma^{2}}{\rho} f_{, \phi}, \quad f_{, \bar{m}}=\overline{f_{, m}} .
$$

The dual tetrad of 1-forms $\left\{\Theta^{(k)}, \Theta^{(l)}, \Theta^{(m)}, \Theta^{(\bar{m})}\right\}$ has a simple form

$$
\begin{gathered}
\Theta^{(k)}=\mathrm{d} v+H \mathrm{~d} u-a, \quad \Theta^{(l)}=\Sigma^{2} \mathrm{~d} u \\
\Theta^{(m)}=\frac{\Sigma}{\sqrt{2}}(\mathrm{~d} \rho-i * \mathrm{~d} \rho), \quad \Theta^{(\bar{m})}=\frac{\Sigma}{\sqrt{2}}(\mathrm{~d} \rho+i * \mathrm{~d} \rho) .
\end{gathered}
$$

With respect to this tetrad, we have found that the non-vanishing curvature components are given by four new components and by those which are the same for the Melvin universe (2.6). The non-vanishing Ricci scalars are

$$
\begin{aligned}
& \Phi_{12}= \frac{1}{4 \sqrt{2}} \frac{1}{\Sigma^{3}}\left(\Sigma^{2} i b_{, m}+2 i b\left(\Sigma^{2}\right)_{, \rho}+2 g_{, m}\right) \\
& \Phi_{22}=\frac{1}{2} \frac{1}{\Sigma^{2}}\left(\Delta H-\left(\Sigma^{-2}\right)_{, \rho} H_{, \rho}+\frac{1}{2} \Sigma^{2} b^{2}\right. \\
&\left.+2 a^{i} g_{, i}+\Sigma^{-2}\left(g+\partial_{u}\right) \operatorname{div}\left(\Sigma^{2} a\right)\right) .
\end{aligned}
$$

\footnotetext{
${ }^{5}$ The terms $\Phi_{22}$ and $\Psi_{4}$ have a little different form which is caused

by the slightly different choice of null tetrad in our paper.
}

and the non-vanishing Weyl scalars read

$$
\begin{aligned}
\Psi_{3} & =\frac{1}{4 \sqrt{2}} \frac{1}{\Sigma^{3}}\left(\Sigma^{2} i b_{, \bar{m}}+i b\left(\Sigma^{2}\right)_{, \rho}+2 g, \bar{m}\right), \\
\Psi_{4} & =\frac{1}{2} \frac{1}{\Sigma^{5}}\left[2 \Sigma a_{\bar{m}} g_{, \bar{m}}+\Sigma\left(H_{, \rho \rho}-\frac{\Sigma^{4}}{\rho^{2}} H_{, \phi \phi}+2 i \frac{\Sigma^{2}}{\rho} H_{, \phi \rho}\right)\right. \\
+ & \Sigma\left(g+\partial_{u}\right)\left(a_{\rho, \rho}-\frac{\Sigma^{4}}{\rho^{2}} a_{\phi, \phi}+i \frac{\Sigma^{2}}{\rho}\left(a_{\rho, \phi}+a_{\phi, \rho}\right)\right) \\
+ & \left(2 \Sigma_{, \rho}-\frac{\Sigma}{\rho}\right)\left(H_{, \rho}+\partial_{u} a_{\rho}+g a_{\rho}\right. \\
& \left.\left.+2 i \frac{\Sigma^{2}}{\rho}\left(H_{, \phi}+\partial_{u} a_{\phi}+g a_{\phi}\right)\right)\right] .
\end{aligned}
$$

In particular, there exists a relation between $\Psi_{3}$ and $\Phi_{12}$ :

$$
\bar{\Phi}_{12}+\Psi_{3}=\frac{1}{4 \sqrt{2}} \frac{1}{\Sigma^{3}}\left[-i\left(\Sigma^{2}\right)_{, \rho} b+4 g, \bar{m}\right] .
$$

Therefore, the metric (2.18) describes the transversal gravitational wave $\left(\Psi_{4}\right.$ term) in the $k$ direction with a longitudal wave component $\left(\Psi_{3}\right.$ term). The gravitational wave is accompanied by an aligned pure radiation field $\left(\Phi_{22}\right.$ term) with non-null component $\left(\Phi_{12}\right.$ term) propagating in the Melvin universe. In fact, the scalars $\Psi_{3}$ and $\Phi_{12}$ are generated by the gyratonic functions $a_{i}$ and the function $g$. In general the spacetime (2.18) is of Petrov type $I I$.

Let us now investigate the subcases of our solutions. When we set the gyratonic functions $a_{i}=0$, the Ricci scalars become

$$
\begin{aligned}
\Phi_{12} & =\frac{1}{2 \sqrt{2}} \frac{1}{\Sigma^{3}} g_{, m}, \\
\Phi_{22} & =\frac{1}{2} \frac{1}{\Sigma^{4} \rho^{2}}\left[\rho\left(\rho H_{, \rho}\right)_{, \rho}+\Sigma^{4} H_{, \phi \phi}\right]
\end{aligned}
$$

and the Weyl scalars then read

$$
\begin{aligned}
\Psi_{3} & =\frac{1}{2 \sqrt{2}} \frac{1}{\Sigma^{3}} g_{, \bar{m}}, \\
\Psi_{4} & =\frac{1}{2} \frac{1}{\Sigma^{5} \rho}\left[\Sigma \rho H_{, \rho \rho}+\left(2 \rho \Sigma_{, \rho}-\Sigma\right) H_{, \rho}\right] \\
& -\frac{1}{2} \frac{1}{\rho^{2}} H_{, \phi \phi}+i \frac{1}{2} \frac{1}{\Sigma^{3} \rho^{2}}\left[2 \partial_{\rho}(\Sigma g H)-3 \Sigma H\right]_{, \phi} .
\end{aligned}
$$

We again obtain the spacetime with similar characteristics as for the full gyratonic metric (2.18). However, the scalars $\Psi_{3}$ and $\Phi_{12}$, which now depend only on function $g$, are now related by an even simpler relation:

$$
\bar{\Phi}_{12}=\Psi_{3} \text {. }
$$

If we assume additionally $H$ to be $v$-independent (i.e., $g=0$ ) we obtain the only non-vanishing scalars $\Phi_{22}$ and $\Psi_{4}$ in the same form as in (5.9) and (5.10). This case and its subcases were thoroughly discussed in [44]. 
Finally, let us mention that for $\varrho_{\mathrm{EM}}=0$ the background reduces to Minkowski spacetime and we thus recover the gyraton moving on the Minkowski background as an important subcase of our solutions.

\section{B. Electromagnetic field}

The gyraton propagates in a non-null electromagnetic field (2.22), the influence of which on the geometry is characterized by its density $\varrho_{\mathrm{EM}}$ (2.4). The electromagnetic field is modified by the gyraton through the $\sigma_{i} \mathrm{~d} u \wedge \mathrm{d} x^{i}$ terms. The electromagnetic field can be rewritten in terms of potentials using (3.24) as

$$
\begin{aligned}
F= & E(\mathrm{~d} v \wedge \mathrm{d} u+\mathrm{d} u \wedge \mathrm{d} \varphi) \\
& +B\left(\Sigma^{-2} \rho \mathrm{d} \rho \wedge \mathrm{d} \phi+\mathrm{d} u \wedge \mathrm{d} \psi\right)
\end{aligned}
$$

It describes a superposition of electric and magnetic fields, both pointing along the $z$ direction, which are modified by the gravitation field of the gyraton. The additional term does not have a simple structure of electric or magnetic field, however both are of the form $\mathrm{d} u \wedge \mathrm{d} f$ with $f$ being the proper potential.

The electromagnetic field projected on the null tetrad (5.2) is characterized by three scalars $\Phi_{i}$,

$$
\Phi_{0}=0, \quad \Phi_{1}=\frac{\overline{\mathcal{B}}}{2 \Sigma^{2}}, \quad \Phi_{2}=\frac{\overline{\mathcal{B}}}{\sqrt{2} \Sigma^{3}}\left[a_{\bar{m}}-\overline{\mathcal{S}}_{\rho}\right]
$$

It follows that the non-null electromagnetic field is aligned with the principal null direction $k$ of the gravitation field, but this vector is not a double degenerate vector of the field.

\section{CONCLUSION}

We have derived and analyzed new gyraton solutions moving with the speed of light on electro-vacuum Melvin background spacetime in four dimensions. This solution extends the gyraton solutions previously known on the Nariai, anti-Nariai, and Plebański-Hacyan universes of type D, and on conformally flat Bertotti-Robinson and Minkowski space.

The gyraton solutions describe a gravitational field created by a stress-energy tensor of a spinning (circularly polarized) high-frequency beam of electromagnetic radiation, neutrino, or any other massless fields. The gyratons generalize standard gravitational $p p$-waves or Kundt waves by admitting a non-zero angular momentum of the source. The interpretation is that the null matter in the interior of the gyratonic source possesses an intrinsic spin (or non-zero angular momentum). This leads to other nontrivial components of the Einstein equations, namely, $G_{u i}$ in addition to the pure radiation $u u$-component which appears for $p p$-waves or Kundt waves.
We have shown that it is possible to define the gyraton by adding the gyratonic terms $a_{i}$ to the gravitational wave on the Melvin spacetime in cylindrical coordinates in a similar way as we have defined them in the general Kundt class. We were able to find an ansatz for the gyraton metric on the Melvin spacetime by direct transformation from the Kundt class of metrics (IIB).

We have further demonstrated that the EinsteinMaxwell equations reduce to the set of linear equations on the 2-dimensional transverse spacetime which has a nontrivial geometry given by the transverse metric. These equations can be solved exactly for any distribution of the matter sources. In general, the problem has been thus reduced to a construction of scalar Green functions for certain differential operators on the transverse space.

We have solved and analyzed the field equations for particular examples with the axial symmetry. In these cases the equations reduce to ordinary differential equations.

We have analyzed geometric properties of the principal null congruence and we have found that it is not recurrent contrary to the case of gyratons on direct product spacetimes. We have explicitly calculated the curvature tensor and determined that the gyratons on Melvin spacetime are of Petrov type II and belong to the Kundt family of shear-free and twist-free nonexpanding spacetimes. The gyratonic term $a_{i}$ generates the non-trivial Ricci $\Phi_{12}$ and Weyl $\Psi_{3}$ scalars, in addition to the gravitational waves investigated in [44]. We found also a very simple relation (5.8) between these components. By studying particular subclasses we have shown that our solutions are generalizations of those from [44].

The scalar polynomial invariants of the metric (2.18) are in general non-constant (although, some of them are zero) - they depend on the coordinate $\rho$. The invariants are not affected by the presence of the gyratons, they are the same as for the Melvin background. The same property was proved for gyratons on backgrounds belonging to VSI or CSI families of spacetimes.

It would be interesting to investigate a generalization of our ansatz for more complicated spacetimes which could allow, e.g., an inclusion of a cosmological constant.

\section{Acknowledgments}

We wish to thank to Tomáš Pecháček and Otakar Svítek for helpful discussions and to Marcello Ortaggio for his paper about gravitational waves in the Melvin universe which motivated our work. H. K. was supported by Grants No. GAČR-202/09/H033, No. GAUK 12209, and Project No. SVV 261301 of the Charles University in Prague. P. K. was supported by Grant No. GAČR 202/09/0772, and both authors thank the Project No. LC06014 of the Center of Theoretical Astrophysics. 


\section{Appendix A: The Einstein equations}

Here we present quantities needed for evaluation of the Einstein equations.

The inverse to the metric (2.18) is

$$
\begin{aligned}
& g^{\mu \nu} \partial_{\mu} \partial_{\nu}=\frac{1}{\Sigma^{2}} \partial_{\rho} \partial_{\rho}+\frac{\Sigma^{2}}{\rho^{2}} \partial_{\phi} \partial_{\phi}-\frac{2}{\Sigma^{2}} \partial_{u} \partial_{v} \\
& \quad+2\left(a_{\rho} \frac{1}{\Sigma^{2}} \partial_{\rho}+a_{\phi} \frac{\Sigma^{2}}{\rho^{2}} \partial_{\phi}\right) \partial_{v}+\left(2 \frac{H}{\Sigma^{2}}+a^{2}\right) \partial_{v} \partial_{v} .
\end{aligned}
$$

The stress-energy tensor $T^{\mathrm{EM}}$ of the electromagnetic field (2.22) can be defined as

$$
T_{\mu \nu}^{\mathrm{EM}}=\frac{\varepsilon_{\mathrm{o}}}{2} \mathcal{F}_{\mu} \rho \overline{\mathcal{F}}_{\nu \rho}
$$

where $\mathcal{F} \equiv F+i \star F$ is the complex self-dual Maxwell tensor. The 4-dimensional Hodge dual is defined by $\star F_{\mu \nu}=\frac{1}{2} \varepsilon_{\mu \nu \rho \sigma} F^{\rho \sigma}$ and the Maxwell tensor $\mathcal{F}_{\mu \nu}$ satisfies the self-duality condition $\star \mathcal{F}=-i \mathcal{F}$.

The non-vanishing components of the stress-energy tensor A2 are

$$
\begin{aligned}
& \varkappa T_{u v}^{\mathrm{EM}}=\frac{\varrho_{\mathrm{EM}}}{\Sigma^{2}}, \\
& \varkappa T_{u u}^{\mathrm{EM}}=2 \varrho_{\mathrm{EM}}\left(\frac{H}{\Sigma^{2}}+(s-a)^{2}\right), \\
& \varkappa T_{u \rho}^{\mathrm{EM}}=\frac{\varrho_{\mathrm{EM}}}{\Sigma^{2}}\left(a_{\rho}-2 s_{\rho}\right), \\
& \varkappa T_{u \phi}^{\mathrm{EM}}=\frac{\varrho_{\mathrm{EM}}}{\Sigma^{2}}\left(a_{\phi}-2 s_{\phi}\right), \\
& \varkappa T_{\rho \rho}^{\mathrm{EM}}=\frac{\varrho_{\mathrm{EM}}}{\Sigma^{2}}=\frac{\varrho_{\mathrm{EM}}}{\Sigma^{4}} g_{\rho \rho}, \\
& \varkappa T_{\phi \phi}^{\mathrm{EM}}=\frac{\varrho_{\mathrm{EM}} \rho^{2}}{\Sigma^{6}}=\frac{\varrho_{\mathrm{EM}}}{\Sigma^{4}} g_{\phi \phi},
\end{aligned}
$$

where the density $\varrho_{\mathrm{EM}}$ was defined in (2.4).

The non-vanishing components of the stress-energy tensor (2.27) of the gyratonic matter are

$$
\begin{aligned}
\varkappa T_{u u}^{\mathrm{gyr}}= & j_{u}=v \operatorname{div}\left(\Sigma^{2} j\right)+\iota, \\
& \varkappa T_{u i}^{\mathrm{gyr}}=j_{i} .
\end{aligned}
$$

The Einstein tensor for the metric (2.18) reads

$$
\begin{aligned}
G_{u v}= & \frac{1}{\Sigma^{2} \rho}\left(-\rho\left(\Sigma_{, \rho}\right)^{2}+2 \Sigma\left(\Sigma_{, \rho}\right)\right), \\
G_{u u}= & \frac{1}{2} \Sigma^{4} b^{2}+\Sigma^{2}\left(\Delta H+\frac{\left(\Sigma^{2}\right)_{, \rho}}{\Sigma^{4}} H_{, \rho}\right)+\Sigma^{2}\left(\partial_{v}^{2} H\right) a^{2} \\
& +2 \Sigma^{2} a^{i} \partial_{v} H_{, i}+\left(\partial_{v} H+\partial_{u}\right) \operatorname{div}\left(\Sigma^{2} a\right) \\
& +2 H G_{u v}, \\
G_{u \rho}= & \frac{1}{2} \frac{\Sigma^{4}}{\rho} b_{, \phi}-a_{\rho}\left(G_{u v}-\partial_{v}^{2} H\right)+\partial_{v} H_{, \rho}, \\
G_{u \phi}= & -\frac{1}{2} \rho\left(b_{, \rho}+\frac{4 \Sigma_{, \rho}}{\Sigma} b\right)-a_{\phi}\left(G_{u v}-\partial_{v}^{2} H\right)+\partial_{v} H_{, \phi}, \\
G_{\rho \rho}= & G_{u v}+\partial_{v}^{2} H, \\
G_{\phi \phi}= & \frac{\rho^{2}}{\Sigma^{4}}\left(G_{u v}+\partial_{v}^{2} H\right) .
\end{aligned}
$$

Here we have used only the metric (2.18), without any usage of the field equations.

\section{Appendix B: The NP formalism}

Calculating the Newman-Penrose spin coefficients with respect to the tetrad (5.2), we recover again that the congruence $k$ is nonexpanding and nontwisting $\left(\rho_{\mathrm{NP}}=0\right)$, sheer-free $\left(\sigma_{\mathrm{NP}}=0\right)$, geodesic and affine parameterized $\left(\kappa_{\mathrm{NP}}=\varepsilon_{\mathrm{NP}}=0\right)$. In addition, the tetrad is gauge invariant and it is not parallelly transported along the null congruence because it does not satisfy $\kappa_{\mathrm{NP}}=\pi_{\mathrm{NP}}=\varepsilon_{\mathrm{NP}}=0$.

The remaining spin coefficients are

$$
\begin{gathered}
\lambda_{\mathrm{NP}}=0, \quad \mu_{\mathrm{NP}}=\frac{i}{2} b \\
\gamma_{\mathrm{NP}}=\frac{1}{4} \frac{1}{\Sigma^{2}}\left(2 g+i \Sigma^{2} b\right), \\
\nu_{\mathrm{NP}}=\frac{1}{\sqrt{2}} \frac{1}{\Sigma^{3}}\left\{\left(g+\partial_{u}\right) a_{\bar{m}}+g, \bar{m}\right\} \\
\tau_{\mathrm{NP}}=-\frac{1}{\sqrt{2}} \frac{1}{\Sigma^{2}} \Sigma_{, \rho}, \quad \pi_{\mathrm{NP}}=+\frac{1}{\sqrt{2}} \frac{1}{\Sigma^{2}} \Sigma_{, \rho} \\
\alpha_{\mathrm{NP}}=\frac{1}{2 \sqrt{2}} \frac{1}{\Sigma^{2} \rho}\left(2 \rho \Sigma_{, \rho}-\Sigma\right), \quad \beta_{\mathrm{NP}}=\frac{1}{2 \sqrt{2}} \frac{1}{\Sigma \rho} .
\end{gathered}
$$

\section{Appendix C: Scalar polynomial curvature invariants}

As we have already mentioned, the scalar curvature invariants are independent of all metric functions which characterize the gyraton, and have the same values as the corresponding invariants of the Melvin universe (cf. [56]). Let us stress here, however, that the invariants are generally non-constant, namely, they depend on the coordinate $\rho$. In this appendix we list some of the curvature invariants.

The scalar curvature for the whole gyraton metric (2.18) is zero, $R=0$. Next, we define the following scalar polynomial invariants constructed from the Riemann tensor:

$$
\begin{aligned}
R^{(2)} & =R^{a b}{ }_{c d} R^{c d}{ }_{a b}, \\
R^{(3)} & =R^{a b}{ }_{c d} R^{c d}{ }_{e f} R^{e f}{ }_{a b}, \\
R^{(4)} & =R^{a b}{ }_{c d} R^{c d}{ }_{e f} R^{e f}{ }_{p q} R^{p q}{ }_{a b}, \\
R^{(5)} & =R^{a b}{ }_{c d} R^{c d}{ }_{e f} R^{e f}{ }_{p q} R^{p q}{ }_{r s} R^{r s}{ }_{a b} .
\end{aligned}
$$

Using the GRtensor package in Maple, we get the explicit 
expressions:

$$
\begin{aligned}
& R^{(2)}= \frac{2 \varrho_{\mathrm{EM}}^{2}}{\Sigma^{8}}\left(\frac{3}{8} \varrho_{\mathrm{EM}}^{2} \rho^{4}-3 \varrho_{\mathrm{EM}} \rho^{2}+10\right) \\
& R^{(3)}=-\frac{3 \varrho_{\mathrm{EM}}^{3}}{\Sigma^{12}}\left(\frac{1}{16} \varrho_{\mathrm{EM}}^{3} \rho^{6}-\frac{3}{4} \varrho_{\mathrm{EM}}^{2} \rho^{4}+7 \varrho_{\mathrm{EM}} \rho^{2}-20\right), \\
& R^{(4)}= \frac{4 \varrho_{\mathrm{EM}}^{4}}{\Sigma^{16}}\left(\frac{9}{256} \varrho_{\mathrm{EM}}^{4} \rho^{8}-\frac{9}{16} \varrho_{\mathrm{EM}}^{3} \rho^{6}\right. \\
&\left.\quad+\frac{51}{8} \varrho_{\mathrm{EM}}^{2} \rho^{4}-33 \varrho_{\mathrm{EM}} \rho^{2}+65\right) \\
& R^{(5)}=-\frac{5 \varrho_{\mathrm{EM}}^{5}}{\Sigma^{20}}\left(\frac{3}{256} \varrho_{\mathrm{EM}}^{5} \rho^{10}-\frac{15}{64} \varrho_{\mathrm{EM}}^{4} \rho^{8}+\frac{31}{8} \varrho_{\mathrm{EM}}^{3} \rho^{6}\right. \\
&\left.\quad-\frac{63}{2} \varrho_{\mathrm{EM}}^{2} \rho^{4}+64 \cdot 127 \varrho_{\mathrm{EM}} \rho^{2}-204\right)
\end{aligned}
$$

We explicitly observe that these invariants do not depend on any of the metric functions $a_{i}, g$, and $h$ which characterize the gyraton.

The invariants (C2) mimic the behavior of the Gauss curvature of the transverse space discussed in detail in (IID). They have their maximum on the axis $\rho=0$ and they are vanishing as "the neck of the vase closes off asymptotically" as $\rho$ tends to infinity. For $\varrho_{\mathrm{EM}}=0$ we get the identically vanishing invariants, i.e., the invariants for the gyratons on Minkowski background (VSI).

In Maple tensor package GRtensor there is defined a set of curvature invariants CMinvars. For completeness, we present the explicit expressions for them:

$$
\begin{gathered}
R=R_{2}=W 1 I=W 2 I=0 \\
R_{1}=\frac{\varrho_{\mathrm{EM}}^{2}}{\Sigma^{8}}, \quad W 1 R=\frac{3 \varrho_{\mathrm{EM}}^{2}}{2^{5} \Sigma^{8}}\left(\varrho_{\mathrm{EM}} \rho^{2}-4\right)^{2}, \\
R_{3}=\frac{\varrho_{\mathrm{EM}}^{4}}{4 \Sigma^{16}}, \quad W 2 R=\frac{3 \varrho_{\mathrm{EM}}^{3}}{2^{8} \Sigma^{12}}\left(\varrho_{\mathrm{EM}} \rho^{2}-4\right)^{3}, \\
M 1 I=M 2 I=M 4=M 5 I=0 \\
M 1 R=\frac{\varrho_{\mathrm{EM}}^{3}}{2^{2} \Sigma^{12}}\left(\varrho_{\mathrm{EM}} \rho^{2}-4\right), \\
M 2 R=M 3=\frac{\varrho_{\mathrm{EM}}^{4}}{2^{4} \Sigma^{16}}\left(\varrho_{\mathrm{EM}} \rho^{2}-4\right)^{2} \\
M 5 R=\frac{\varrho_{\mathrm{EM}}^{5}}{2^{6} \Sigma^{20}}\left(\varrho_{\mathrm{EM}} \rho^{2}-4\right)^{3} .
\end{gathered}
$$

[1] R. C. Tolman, Relativity, Thermodynamics, and Cosmology (Clarendon Press, Oxford, 1934).

[2] A. Peres, Phys. Rev. 118, 1105 (1960).

[3] W. B. Bonnor, Commun. Math. Phys. 13, 163 (1969).

[4] W. B. Bonnor, Int. J. Theor. Phys. 2, 373 (1969).

[5] W. B. Bonnor, Int. J. Theor. Phys. 3, 57 (1970).

[6] H. Stephani, D. Kramer, M. Maccallum, C. Hoenselaers, and E. Herlt, Exact Solutions of Einstein's Field Equations (Cambridge University Press, Cambridge, 2003).

[7] J. B. Griffiths and J. Podolský, Exact Space-Times in Einstein's General relativity (Cambridge University Press, Cambridge, 2009).

[8] P. C. Aichelburg and R. U. Sexl, Gen. Rel. Grav. 2, 303 (1971).

[9] V. Ferrari and P. Pendenza, Gen. Rel. Grav. 22, 1105 (1990).

[10] C. O. Loustó and N. Sánchez, Nucl. Phys. B 383, 377 (1992).

[11] M. Hotta and M. Tanaka, Class. Quant. Gravity 10, 307 (1993).

[12] H. Balasin and H. Nachbagauer, Class. Quant. Gravity 12, 707 (1995).

[13] H. Balasin and H. Nachbagauer, Class. Quant. Gravity 13, 731 (1996).

[14] J. Podolský and J. B. Griffiths, Phys. Rev. D 56, 4756 (1997).

[15] J. Podolský and J. B. Griffiths, Phys. Rev. D 58, 124024 (1998).

[16] J. Podolský, in Gravitation: Following the Prague Inspiration, edited by O. Semerák, J. Podolský, and M. Žofka (World Scientific, Singapore, 2002), pp. 205-246, grqc/0201029.

[17] C. Barrabès and P. A. Hogan, Singular null hypersurfaces in general relativity (World Scientific, Singapore, 2003).

[18] W. B. Bonnor, Int. J. Theor. Phys. 3, 257 (1970).
[19] J. B. Griffiths, Int. J. Theor. Phys. 5, 141 (1972a).

[20] H. W. Brinkmann, Math. Ann. 94, 119 (1925).

[21] V. P. Frolov and D. V. Fursaev, Phys. Rev. D 71, 104034 (2005).

[22] V. P. Frolov, W. Israel, and A. Zelnikov, Phys. Rev. D 72, 084031 (2005).

[23] V. Pravda, A. Pravdova, A. Coley, and R. Milson, Class. Quant. Gravity 19, 6213 (2002).

[24] D. N. Page, Class. Quant. Gravity 26, 055016 (2009).

[25] V. P. Frolov and A. Zelnikov, Class. Quant. Gravity 23, 2119 (2006).

[26] V. P. Frolov and A. Zelnikov, Phys. Rev. D 72, 104005 (2005).

[27] S. T. C. Siklos, Galaxies, Axisymmetric Systems and Relativity (Cambridge University Press ed M. A. H. MacCallum, Cambridge, 1985).

[28] J. Podolský, Class. Quant. Gravity 15, 719 (1998).

[29] A. A. Coley, S. Hervik, and N. Pelavas, Class. Quant. Gravity 23, 3053 (2006).

[30] A. A. Coley, G. W. Gibbons, S. Hervik, and C. N. Pope, Class. Quant. Gravity 25, 145017 (2008).

[31] A. A. Coley, S. Hervik, and N. Pelavas, Class. Quant. Gravity 25, 025008 (2008).

[32] A. A. Coley, S. Hervik, and N. Pelavas, Class. Quant. Gravity 26, 025013 (2009).

[33] H. Kadlecová, A. Zelnikov, P. Krtouš, and J. Podolský, Phys. Rev. D 80, 024004 (2009).

[34] V. P. Frolov and F.-L. Lin, Phys. Rev. D 73, 104028 (2006).

[35] M. M. Caldarelli, D. Klemm, and E. Zorzan, Class. Quant. Gravity 24, 1341 (2007).

[36] H. Yoshino and V. S. Rychkov, Phys. Rev. D 71, 104028 (2005).

[37] H. Yoshino and R. B. Mann, Phys. Rev. D 74, 044003 (2006). 
[38] H. Yoshino, A. Zelnikov, and V. Frolov, Phys. Rev. D 75, 124005 (2007).

[39] W. B. Bonnor, Proc. Phys. Soc. London A 67, 225 (1954).

[40] M. A. Melvin, Phys. Rev. 139, B225 (1965).

[41] K. S. Thorne, Phys. Rev. 139, B244 (1965).

[42] Melvin and Wallingford, J. Math. Phys. (N.Y.) 7, 333 (1966).

[43] D. Garfinkle and M. A. Melvin, Phys. Rev. D 45, 1188 (1992).

[44] M. Ortaggio, Phys. Rev. D 69, 064034 (2004).

[45] L. Havrdová and P. Krtouš, Gen. Rel. Grav. 39, 291 (2007).

[46] G. W. Gibbons and K. Maeda, Nucl. Phys. B 298, 741 (1988).

[47] G. W. Gibbons and C. A. R. Herdeiro, Class. Quant. Gravity 18, 1677 (2001).

[48] G. W. Gibbons, in Fields and Geometry: Proceedings of the XXII Winter School of Theoretical Physics, edited by A. Jadczyk (World Scientific, Singapore, 1986).

[49] D. Garfinkle and A. Strominger, Phys. Rev. Lett. B 256, 146 (1991).

[50] D. Garfinkle, S. B. Giddings, and A. Strominger, Phys. Rev. D 49, 958 (1994).

[51] F. Dowker, J. P. Gauntlett, D. A. Castor, and J. Traschen, Phys. Rev. D 49, 2909 (1994).

[52] F. Dowker, J. P. Gauntlett, S. B. Giddings, and G. T. Horowitz, Phys. Rev. D 50, 2662 (1994).

[53] S. W. Hawking and S. Ross, Phys. Rev. D 52, 5865 (1995).

[54] R. Emparan, Phys. Rev. Lett. 75, 3386 (1995).

[55] J. F. Plebanski, J. Math. Phys. 20, 1946 (1979).

[56] A. A. Coley, S. Hervik, and N. Pelavas, Class. Quant. Gravity 27, 102001 (2010). 